\title{
Uniqueness of Self-Similar Solutions for a Crystalline Flow
}

\author{
Alina Stancu
}

1. Introduction. In his paper, "Evolving plane curves by curvature in relative geometries," M. Gage shows that, under certain technical assumptions, every smooth weighted curvature flow is the curve-shortening flow of a unique Minkowski geometry. Moreover, each Minkowski curvature flow has a unique self-similar solution $([9])$.

The goal of this paper is to use the analogy between the motion of convex, polygonal curves by crystalline curvature and the motion of smooth, convex curves by curvature in the Minkowski geometry to prove the uniqueness of selfsimilar solutions for a crystalline flow.

The motion by crystalline curvature was independently defined by M. Gurtin and J. E. Taylor ([1], [14], [19]). Taylor defines the crystalline curvature flow so that the normal velocity of each segment is inversely proportional to the length of that segment where the proportionality factor is determined by the Wulff shape $\tilde{K}$ as in (1). Our paper settles the uniqueness of self-similar solutions and the asymptotic behaviour of solutions, as long as the Wulff shape is symmetric with respect to the origin.

To be precise, let $K$ and $\tilde{K}$ be planar, convex polygons with the same number of parallel sides, $n>4$. Let $\ell_{i}$ and $\tilde{\ell}_{i}$ be the lengths of the corresponding parallel sides, of $K$ and $\tilde{K}$, and let $h_{i}$ and $\tilde{h}_{i}$ be the respective distances of each side from the origin. Under the crystalline flow each boundary segment of $K$ will flow inward at a rate given by:

$$
\frac{\partial h_{i}}{\partial t}=-\frac{\tilde{h}_{i} \tilde{\ell}_{i}}{\ell_{i}} \quad \text { for all } \quad 1 \leq i \leq n .
$$

It is known that this flow is defined and continous for any polygonal curve, up until the time that all line segments have zero length ([19]). J. E. Taylor conjectured that the only self-similar solutions $K$ of (1) are homotheties of $\tilde{K}$, provided that $\tilde{K}$ is not a parallelogram $([18])$. 
We prove here, under a symmetry assumption, J. E. Taylor's conjecture. In the process we prove a much stronger result: any admissible polygonal convex curve flowing by (1) will shrink uniformly to a $\tilde{K}$-shaped point.

Our main result is as follows:

Theorem 1.1 If $n>4$ and $\tilde{K}$ is centrally symmetric, then the only selfsimilar solution of (1) is a homothety of $\tilde{K}$ and this is an attractor of any other solution.

It is easy to show that parallelograms are a special case and that for $n=4$ there are many self-similar solutions. For $n=3$ all triangles with corresponding parallel sides are self-similar.

The most natural deformation by curvature of a smooth, closed curve occurs for the curve-shortening equation ([8], [11], [13]). This is the isotropic version of the weighted curvature flow. A curve evolving by weighted curvature has its normal velocity depending also on a positive 'weight' function defined on the set of unitary directions. This motion has been studied by many authors in a variety of contexts $([5],[7],[9],[12])$.

M.Gage's approach proved that every anisotropic curvature flow on embedded, closed curves for which the weight function is smooth, symmetric and strictly positive arises as a "gradient" flow for the length functional of a unique Minkowski geometry ([9], [12]). Every embedded, closed curve will shrink to a point with its shape approaching the shape of the isoperimetrix of the associated Minkowski geometry. This implies that the shape of the isoperimetrix is the unique self-similar solution of the flow $([9])$.

Our result also has implications in the field of material sciences. In this setting, the curve is interpreted as an evolving free boundary separating a two dimensional two-phase system. The weight function is the energy density which endows the curve with energy and determines what is known as the Wulff shape. The Wulff shape has the least possible boundary energy compared to all other curves enclosing the same area. In the Minkowski geometry the boundary of the Wulff shape is known as the isoperimetrix because it minimizes the Minkowski boundary length for a fixed enclosed area.

It is of a natural interest in material sciences to consider interfacial energy densities that are continous, but not smooth. These crystalline energies correspond to polygonal Wulff shapes and it is precisely the flows associated to these energies which we consider in this article.

A few problems remain open. One is the uniqueness of self-similar solutions if the assumption on the symmetry of $\tilde{K}$ is removed. In fact, this is still unaswered even in the smooth case of the flow of curves by weighted curvature. On the other hand, the results of this paper can be proved for a larger class of rectifiable curves that combine flat sides with parts of strictly positive, finite curvature, as long as we require $K$ to have the flat sides parallel to flat sides of the reference shape $\tilde{K}$. The question of the existence of solutions of such flows is also being studied. ([17])

The paper is organized as follows. In Section 2 we present the basic definitions from convex geometry. We continue in Section 3 by calculating the 
evolution equations for the geometric quantities defined in the previous section, extending Gage's results to piecewise-smooth, convex, but not strictly convex, bodies. The last section concludes the proof of the main result. It is here, in Theorem 4.2 , that the symmetry of $\tilde{K}$ and the fact that the number of sides of $\tilde{K}$ is greater than four play their crucial role.

This work is part of the author's doctoral dissertation.

\section{Elements of convex geometry}

Definition 2.1 A solution to (1) is geometrically self-similar if it is the boundary of a convex body which evolves without changing shape; i.e. $K(t)=$ $\lambda(t) \bar{K}$ for some fixed compact body $\bar{K}$.

Recall that the support function of a convex body $K$ is defined as $h(u)=$ $\sup \{\langle x, u\rangle \mid x \in K\}$, on all the unitary directions $u \in \mathbf{R}^{2}$. One often considers the restriction of $h$ to the unit circle, $h(\vartheta)=h(u(\vartheta))=h\left(e_{1} \cos (\vartheta)+e_{2} \sin (\vartheta)\right)$, where $\left\{e_{1}, e_{2}\right\}$ is an orthonormal basis of $\mathbf{R}^{2}$. If the support function of a convex planar curve is of class $\mathbf{C}^{2}$ and $k$ is its curvature function, then: $1 / k=h+h_{\vartheta \vartheta}$.

So, for each $\vartheta$, there exists a unitary vector $e(\vartheta)$ in the exterior normal cone to $K$ such that the supremum is reached: $h(\vartheta)=\langle X(\vartheta), e(\vartheta)\rangle$, where $X(\vartheta)=$ the position function of $K$ in the direction $u(\vartheta)$.

In particular, on a polygonal curve $K$,

$$
h_{i}=\operatorname{dist}\left(0, \ell_{i}\right)=h\left(\vartheta_{i}\right)=\left\langle X\left(\vartheta_{i}\right), e\left(\vartheta_{i}\right)\right\rangle,
$$

where $\vartheta_{i}$ is the angle corresponding to the normal direction to the $i$-th side of $K$.

In fact, for any polygon, $K$, we obtain a continous support function, smooth everywhere except on the set $\left\{\vartheta_{i}\right\}_{i=1}^{n}$, corresponding to the flat sides of $K$, of length $\ell_{i}$, where

$$
h_{\vartheta}^{+}\left(\vartheta_{i}\right)-h_{\vartheta}^{-}\left(\vartheta_{i}\right)=\ell_{i}, i=1, \ldots, n, \text { with } h_{\vartheta}^{ \pm}\left(\vartheta_{i}\right)=\lim _{\vartheta \rightarrow \vartheta_{i}^{ \pm}} \frac{h(\vartheta)-h\left(\vartheta_{i}\right)}{\vartheta-\vartheta_{i}} .
$$

Remark. The formula (1) in the form:

$$
\left(\frac{h_{i}}{\tilde{h}_{i}}\right)_{t}=-\frac{\tilde{\ell}_{i}}{\ell_{i}}
$$

resembles strongly the symmetric form of the anisotropic curvature flow of curves in the Minkowski geometry as defined by M. Gage:

$$
\left(\frac{h}{\tilde{h}}\right)_{t}=-\frac{k}{\tilde{k}}
$$

where $h, \tilde{h}:[0,2 \pi] \longrightarrow \mathbf{R}_{+}$are the support functions of $K$ and $\tilde{K}$, while $k$, $\tilde{k}:[0,2 \pi] \longrightarrow \mathbf{R}_{+}$are, respectively, the curvature functions for the two bodies. 
We can now extend the definition of the flow to the vertices of the polygon:

Lemma 2.1 The speed of the vertices is completely determined by the flow of the adjacent sides:

$$
\left(X_{v_{i}}\right)_{t}=\frac{d}{d t}\left[\left(h_{i}-h_{i+1}\right)^{2}+4 h_{i} h_{i+1} \cos ^{2}\left(\alpha_{i} / 2\right)\right]^{1 / 2} \cdot\left|\csc \left(\alpha_{i}\right)\right|,
$$

where $\alpha_{i}$ is the angle of the polygon at the $i$-th vertex, $v_{i}$.

Proof.

$$
X_{v_{i}}=\frac{h_{i}}{\cos (\beta)}=\frac{h_{i+1}}{\cos \left(\pi-\alpha_{i}-\beta\right)},
$$

where $\beta$ is the angle between $X_{v_{i}}$ and $h_{i}$. So:

$$
\tan (\beta)=\left(\frac{h_{i+1}}{h_{i}}+\cos \left(\alpha_{i}\right)\right) \cdot \csc \left(\alpha_{i}\right)
$$

and since

$$
\sec ^{2}(\beta)=1+\tan ^{2}(\beta)
$$

the formula (2) follows.

We now define the following geometric quantities associated to a pair $(K, \tilde{K})$ of polygons with parallel corresponding sides.

For a given $\tilde{K}$, the mixed volume of $\mathbf{K}$ and $\tilde{\mathbf{K}}$ is

$$
\mathbf{L}=\sum_{i=1}^{n} \int_{\vartheta_{i}}^{\vartheta_{i+1}}\left(h \tilde{h}-h_{\vartheta} \tilde{h}_{\vartheta}\right) d \vartheta
$$

where $\vartheta_{n+1}=\vartheta_{1}$. The integration by parts formula, combined with the fact that for a polygon we have $h+h_{\vartheta \vartheta}=0$ away from the $\vartheta_{i}$ directions, gives two more alternate forms of the mixed volume:

$$
\begin{aligned}
\mathbf{L} & =\sum_{i=1}^{n} \int_{\vartheta_{i}}^{\vartheta_{i+1}}\left(\tilde{h}+\tilde{h}_{\vartheta \vartheta}\right) h d \vartheta-\left.\sum_{i=1}^{n} \tilde{h}_{\vartheta} h\right|_{\vartheta_{i}} ^{\vartheta_{i+1}} \\
& =-\left.\sum_{i=1}^{n} \tilde{h}_{\vartheta}^{-} h\right|_{\vartheta=\vartheta_{i+1}}+\left.\sum_{i=1}^{n} \tilde{h}_{\vartheta}^{+} h\right|_{\vartheta=\vartheta_{i}} \\
& =\left.\sum_{i=1}^{n}\left(\tilde{h}_{\vartheta}^{+}-\tilde{h}_{\vartheta}^{-}\right) h\right|_{\vartheta=\vartheta_{i}}=\sum_{i=1}^{n} h_{i} \tilde{\ell}_{i}
\end{aligned}
$$

And, similarly,

$$
\mathbf{L}=\sum_{i=1}^{n} \tilde{h}_{i} \ell_{i}
$$


The definition of the area of $\mathbf{K}$ is

$$
\begin{aligned}
\mathbf{A} & =\frac{1}{2} \sum_{i=1}^{n} \int_{\vartheta_{i}}^{\vartheta_{i+1}}\left(h^{2}-h_{\vartheta}^{2}\right) d \vartheta \\
& =\frac{1}{2} \sum_{i=1}^{n} \int_{\vartheta_{i}}^{\vartheta_{i+1}} h\left(h+h_{\vartheta \vartheta}\right) d \vartheta-\left.\frac{1}{2} \sum_{i=1}^{n} h h_{\vartheta}\right|_{\vartheta_{i}} ^{\vartheta_{i+1}} \\
& =\frac{1}{2} \sum_{i=1}^{n} h_{i} \ell_{i} .
\end{aligned}
$$

Note that, since $h+h_{\vartheta \vartheta}=\tilde{h}+\tilde{h}_{\vartheta \vartheta}=0$ for all $\vartheta \neq \vartheta_{i}$, we regain in (4) the ordinary definition of the area obtained by carving the polygon into triangles.

Also, observe that the area of $K$ is defined as half of the mixed volume of $K$ with itself.

Definition 2.2 An annulus of $K$ relative to $\tilde{K}$, of center $O$, inner radius $\rho_{\text {in }}$ and outer radius $\rho_{\text {out }}$, is the set of all the homotheties of $\tilde{K}$, centered at $O$, of factor $\rho, \quad \rho_{\text {in }} \leq \rho \leq \rho_{\text {out }}$, such that $\rho_{\text {in }} \tilde{K} \subseteq K$ and $K \subseteq \rho_{\text {out }} \tilde{K}$. The minimal annulus of $K$ with respect to $\tilde{K}$ is the one which minimizes $\rho_{\text {out }}-\rho_{\text {in }}$.

3. Evolution equations. Throughout this section the following is assumed to be true: There exists a choice of the origin such that $\mathbf{B}_{K}(\rho) \geq 0$ for $\rho_{\text {in }} \leq \rho \leq$ $\rho_{\text {out }}$, where $\mathbf{B}_{K}$ is the Bonessen functional of $K$ with respect to $\tilde{K}, \mathbf{B}_{K}(\rho)=$ $\rho \mathbf{L}-\mathbf{A}-\rho^{2} \alpha, \alpha=\operatorname{Area}(\tilde{K})$ and $\rho_{\text {in }}\left(\rho_{\text {out }}\right)$ are the inner (the outer) radius, respectively, of the minimal annulus of $K$ relative to $\tilde{K}$. Moreover, this origin is the center of the minimal annulus of $K$ and $\tilde{K}$. This will be substantiated in the last section.

The motion of polygonal curves by crystalline curvature is analogous to the motion of curves by curvature in the Minkowski geometry, not only in that the normal velocity is proportional to the crystalline analog of curvature, but also in that certain geometric quantities satisfy similar behaviour in their evolution.

$$
\begin{aligned}
\mathbf{L}_{t} & =\sum_{i=1}^{n} \int_{\vartheta_{i}}^{\vartheta_{i+1}}\left(h_{t} \tilde{h}-h_{\vartheta t} \tilde{h}_{\vartheta}\right) d \vartheta \\
& =\sum_{i=1}^{n} \int_{\vartheta_{i}}^{\vartheta_{i+1}} h_{t}\left(\tilde{h}+\tilde{h}_{\vartheta \vartheta}\right) d \vartheta-\left.\sum_{i=1}^{n} \tilde{h}_{\vartheta} h_{t}\right|_{\vartheta_{i}} ^{\vartheta_{i+1}} \\
& =-\sum_{i=1}^{n} \frac{\tilde{h}_{i} \tilde{\ell}_{i}^{2}}{\ell_{i}}
\end{aligned}
$$


And:

$$
\begin{aligned}
\mathbf{A}_{t} & =\frac{1}{2} \sum_{i=1}^{n} \int_{\vartheta_{i}}^{\vartheta_{i+1}}\left(2 h h_{t}-2 h_{\vartheta} h_{\vartheta t}\right) d \vartheta \\
& =\sum_{i=1}^{n} \int_{\vartheta_{i}}^{\vartheta_{i+1}}\left(h+h_{\vartheta \vartheta}\right) h_{t} d \vartheta-\left.\sum_{i=1}^{n} h_{\vartheta} h_{t}\right|_{\vartheta_{i}} ^{\vartheta_{i+1}} \\
& =-\sum_{i=1}^{n} \tilde{h}_{i} \tilde{\ell}_{i}=-2 \alpha
\end{aligned}
$$

Therefore we have proved the following result:

Lemma $\mathbf{3 . 1}$ : The mixed volume $\mathbf{A}$ decreases at a constant rate, proportional to the mixed volume of the reference body.

Proposition 3.1 The mixed volume ratio $\mathbf{L}^{2} / \mathbf{A}$ is decreasing.

Proof.

$$
\begin{aligned}
\left(\frac{\mathbf{L}^{2}}{\mathbf{A}}\right)_{t} & =\frac{2 \mathbf{L} \mathbf{L}_{t}}{\mathbf{A}}-\frac{\mathbf{L}^{2}}{\mathbf{A}^{2}} \cdot \mathbf{A}_{t}=\frac{\mathbf{L}}{\mathbf{A}} \cdot\left(2 \mathbf{L}_{t}-\frac{\mathbf{L}}{\mathbf{A}} \cdot(-2 \alpha)\right) \\
& =-2 \frac{\mathbf{L}}{\mathbf{A}}\left(\sum_{i=1}^{n} \frac{\tilde{h}_{i} \tilde{\ell}_{i}^{2}}{\ell_{i}}-\frac{\mathbf{L} \cdot \alpha}{\mathbf{A}}\right) .
\end{aligned}
$$

Notice that by the CBS-inequality:

$$
\mathbf{L}^{2}=\left(\sum_{i=1}^{n} h_{i} \tilde{\ell}_{i}\right)^{2} \leq \sum_{i=1}^{n}\left(\frac{h_{i}}{\tilde{h}_{i}}\right)^{2} \tilde{h}_{i} \ell_{i} \cdot \sum_{i=1}^{n}\left(\frac{\tilde{\ell}_{i}}{\ell_{i}}\right)^{2} \tilde{h}_{i} \ell_{i}
$$

This implies:

$$
\sum_{i=1}^{n}\left(\frac{\tilde{\ell}_{i}}{\ell_{i}}\right)^{2} \tilde{h}_{i} \ell_{i} \geq \frac{\mathbf{L}^{2}}{\sum_{i=1}^{n}\left(\frac{h_{i}}{\tilde{h}_{i}}\right)^{2} \tilde{h}_{i} \ell_{i}}
$$

Recall that there exists a choice of the origin such that $\mathbf{B}_{K}(h / \tilde{h}) \geq 0$ for all $\vartheta$ 's. For this choice of the origin we have:

$$
\begin{aligned}
0 & \leq \sum_{i=1}^{n} \mathbf{B}_{K}\left(\frac{h_{i}}{\tilde{h}_{i}}\right) \tilde{h}_{i} \ell_{i}=\sum_{i=1}^{n}\left[\frac{h_{i}}{\tilde{h}_{i}} \mathbf{L}-\mathbf{A}-\left(\frac{h_{i}}{\tilde{h}_{i}}\right)^{2} \alpha\right] \tilde{h}_{i} \ell_{i} \\
& =\mathbf{L} \cdot \sum_{i=1}^{n} h_{i} \ell_{i}-\mathbf{A} \cdot \sum_{i=1}^{n} \tilde{h}_{i} \ell_{i}-\alpha \cdot \sum_{i=1}^{n}\left(\frac{h_{i}}{\tilde{h}_{i}}\right)^{2} \tilde{h}_{i} \ell_{i} \\
& =\mathbf{A} \mathbf{L}-\alpha \cdot \sum_{i=1}^{n}\left(\frac{h_{i}}{\tilde{h}_{i}}\right)^{2} \tilde{h}_{i} \ell_{i} .
\end{aligned}
$$


Therefore, by ( 7$)$ and ( 8 ):

$$
\begin{aligned}
\left(\frac{\mathbf{L}^{2}}{\mathbf{A}}\right)_{t} & =-2 \frac{\mathbf{L}}{\mathbf{A}}\left(\sum_{i=1}^{n} \frac{\tilde{h}_{i} \tilde{\ell}_{i}^{2}}{\ell_{i}}-\frac{\mathbf{L} \cdot \alpha}{\mathbf{A}}\right) \\
& \leq-2 \frac{\mathbf{L}}{\mathbf{A}}\left(\frac{\mathbf{L}^{2}}{\sum_{i=1}^{n}\left(\frac{h_{i}}{\tilde{h}_{i}}\right)^{2} \tilde{h}_{i} \ell_{i}}-\frac{\mathbf{L} \cdot \alpha}{\mathbf{A}}\right) \leq 0 .
\end{aligned}
$$

Following M. Gage [9], we now prove the following result:

Lemma 3.2 Let $0<t<T$ be the maximal interval on which the solution to the flow exists.

Then:

$$
\liminf _{t \rightarrow T}\left\{\mathbf{L} \sum_{i=1}^{n}\left(\frac{\tilde{\ell}_{i}}{\ell_{i}}\right)^{2} \tilde{h}_{i} \ell_{i}-\frac{\mathbf{L}^{2} \alpha}{\mathbf{A}}\right\}=0 .
$$

Proof. We have proved that:

$$
\left\{\mathbf{L} \sum_{i=1}^{n}\left(\frac{\tilde{\ell}_{i}}{\ell_{i}}\right)^{2} \tilde{h}_{i} \ell_{i}-\frac{\mathbf{L}^{2} \alpha}{\mathbf{A}}\right\} \geq 0 .
$$

Suppose that:

$$
\liminf _{t \rightarrow T}\left\{\mathbf{L} \sum_{i=1}^{n}\left(\frac{\tilde{\ell}_{i}}{\ell_{i}}\right)^{2} \tilde{h}_{i} \ell_{i}-\frac{\mathbf{L}^{2} \alpha}{\mathbf{A}}\right\} \geq \varepsilon>0 .
$$

Then:

$$
\left(\frac{\mathbf{L}^{2}}{\mathbf{A}}\right)_{t} \leq-\frac{2 \varepsilon}{\mathbf{A}}
$$

Recall that: $\mathbf{A}(t)=\mathbf{A}(0)-2 \alpha t$ and since $\mathbf{A}(T)=0 \quad \Rightarrow \quad \mathbf{A}(0)-2 \alpha T=0$, $\mathbf{A}(t)=2 \alpha(T-t)$.

In other words,

$$
\left(\frac{\mathbf{L}^{2}}{\mathbf{A}}\right)_{t} \leq-\frac{\varepsilon}{\alpha(T-t)}
$$

It follows then:

$$
\int_{0}^{T}\left(\frac{\mathbf{L}^{2}}{\mathbf{A}}\right)_{t} d t \leq-\int_{0}^{T} \frac{\varepsilon}{\alpha(T-t)} d t
$$


and

$$
\left.\left(\frac{\mathbf{L}^{2}}{\mathbf{A}}\right)\right|_{0} ^{T} \leq \frac{\varepsilon}{\alpha} \ln (T-t)=\frac{\varepsilon}{\alpha}(\ln 0-\ln T)=-\infty,
$$

which contradicts the Minkowski inequality $\mathbf{L}^{2} / \mathbf{A} \geq 4 \alpha$.

Lemma 3.3 Let $\tilde{K}$ be symmetric with respect to the origin. There is a positive functional, $\delta(K)$, associated to any convex polygon $K$ such that if the origin is chosen to be the center of the minimal annulus of $K$ relative to $\tilde{K}$, $\left(\mathbf{L}^{2} / \mathbf{A}\right)_{t} \equiv 0$ implies $\delta(K) \equiv 0$.

Proof. Observe that $\mathbf{B}_{K}$ is concave, so it lies above its secant lines, hence:

$$
\mathbf{B}_{K}(x) \geq \mathbf{B}_{K}\left(\rho_{\mathrm{in}}\right)+\frac{\mathbf{B}_{K}\left(\rho_{\text {out }}\right)-\mathbf{B}_{K}\left(\rho_{\mathrm{in}}\right)}{\rho_{\text {out }}-\rho_{\text {in }}} \cdot\left(x-\rho_{\text {in }}\right) .
$$

So:

$$
\mathbf{B}_{K}(x) \geq \frac{\left(x-\rho_{\text {in }}\right)}{\rho_{\text {out }}-\rho_{\text {in }}} \cdot \mathbf{B}_{K}\left(\rho_{\text {out }}\right)+\frac{\left(\rho_{\text {out }}-x\right)}{\rho_{\text {out }}-\rho_{\text {in }}} \cdot \mathbf{B}_{K}\left(\rho_{\text {in }}\right) \geq 0
$$

for any $x \in\left[\rho_{\text {in }}, \rho_{\text {out }}\right]$.

Therefore,

$$
\begin{aligned}
& \sum_{i=1}^{n} \mathbf{B}_{K}\left(\frac{h_{i}}{\tilde{h}_{i}}\right) \tilde{h}_{i} \ell_{i} \\
\geq & \frac{\mathbf{B}_{K}\left(\rho_{\text {out }}\right)-\mathbf{B}_{K}\left(\rho_{\text {in }}\right)}{\rho_{\text {out }}-\rho_{\text {in }}} \cdot \sum_{i=1}^{n} h_{i} \ell_{i}+\frac{\rho_{\text {out }} \cdot \mathbf{B}_{K}\left(\rho_{\text {in }}\right)-\rho_{\text {in }} \cdot \mathbf{B}_{K}\left(\rho_{\text {out }}\right)}{\rho_{\text {out }}-\rho_{\text {in }}} \cdot \sum_{i=1}^{n} \tilde{h}_{i} \ell_{i} \\
= & 2 \mathbf{A} \cdot \frac{\mathbf{B}_{K}\left(\rho_{\text {out }}\right)-\mathbf{B}_{K}\left(\rho_{\text {in }}\right)}{\rho_{\text {out }}-\rho_{\text {in }}}+\mathbf{L} \cdot \frac{\rho_{\text {out }} \cdot \mathbf{B}_{K}\left(\rho_{\text {in }}\right)-\rho_{\text {in }} \cdot \mathbf{B}_{K}\left(\rho_{\text {out }}\right)}{\rho_{\text {out }}-\rho_{\text {in }}} \\
= & 2 \mathbf{A L}-2 \mathbf{A}\left(\rho_{\text {out }}+\rho_{\text {in }}\right) \alpha-\mathbf{A L}+\rho_{\text {in }} \rho_{\text {out }} \mathbf{L} \alpha \\
= & \mathbf{A L} \cdot\left(1+\alpha \cdot \frac{\rho_{\text {in }} \rho_{\text {out }}}{\mathbf{A}}-2 \alpha \cdot \frac{\rho_{\text {out }}+\rho_{\text {in }}}{\mathbf{L}}\right) \\
= & : \mathbf{A L} \cdot \delta(K) .
\end{aligned}
$$

Notice that $\delta(K)$ defined as above is invariant with respect to translations and homotheties and is a continous function of convex sets using the Hausdorff metric. So if the origin $O$ is chosen to be the center of the minimal annulus, we have $\mathbf{B}_{\mathbf{K}}(\rho) \geq 0$ for all $\rho \in\left[\rho_{\text {in }}, \rho_{\text {out }}\right]$. Then by $(11), \delta(K) \geq 0$. 
Now choose $\rho=h / \tilde{h}$ as in the previous lemma and consider

$$
\begin{aligned}
0 & \leq \mathbf{A} \mathbf{L} \cdot \delta(K) \leq \sum_{i=1}^{n} \mathbf{B}_{K}\left(\frac{h_{i}}{\tilde{h}_{i}}\right) \tilde{h}_{i} \ell_{i} \\
& =\mathbf{L} \cdot\left(\sum_{i=1}^{n} h_{i} \ell_{i}\right)-\mathbf{A} \cdot\left(\sum_{i=1}^{n} \tilde{h}_{i} \ell_{i}\right)-\alpha \cdot\left[\sum_{i=1}^{n}\left(\frac{h_{i}}{\tilde{h}_{i}}\right)^{2} \tilde{h}_{i} \ell_{i}\right] \\
& =2 \mathbf{A} \mathbf{L}-\mathbf{A} \mathbf{L}-\alpha \cdot\left[\sum_{i=1}^{n}\left(\frac{h_{i}}{\tilde{h}_{i}}\right)^{2} \tilde{h}_{i} \ell_{i}\right] .
\end{aligned}
$$

It follows then:

$$
\frac{\mathbf{A L}}{\alpha}(1-\delta(K)) \geq \sum_{i=1}^{n}\left(\frac{h_{i}}{\tilde{h}_{i}}\right)^{2} \tilde{h}_{i} \ell_{i}
$$

which concludes:

$$
\left(\frac{\mathbf{L}^{2}}{\mathbf{A}}\right)_{t} \equiv 0 \quad \Rightarrow \delta(K)=0
$$

To prove the assumption of the section 3 , and to conclude our result by showing that $\delta(K)=0$ if and only if $K$ is a translate of a homothetic transformation of $\tilde{K}$, we consider the theorem 4.2. The version for smooth bodies is due to M. Gage [9].

4. Proof of the Main Theorem. Let $\mathbf{B}_{K}(\rho)=\rho L-A-\alpha \rho^{2}$ be the Bonnesen functional of a convex body $K$ with respect to the reference body $\tilde{K}$.

If we denote by $r_{\text {in }}$ the inner radius and by $r_{\text {out }}$ the outer radius of $K$ with respect to $\tilde{K}$, then the following is true:

Theorem 4.1 (Blaschke [2] or Flanders [6], or Bonnesen in the Euclidean case [3] )

$$
\mathbf{B}_{K}(\rho) \geq 0 \text { for all } \rho \in\left[r_{\text {in }}, r_{\text {out }}\right] .
$$

Also, if both $K$ and $\tilde{K}$ are smooth, $\mathbf{B}_{K} \equiv 0$ implies either $r_{\mathrm{in}}=r_{\text {out }}$ and $K=\tilde{K}$ or $\rho=r_{\text {in }}$ and $K$ is the Minkowski sum of a line and $\tilde{K}$. If $r_{\text {out }} \geq \rho>$ $r_{\text {in }}$, then $\mathbf{B}_{K}(\rho)$ cannot be zero.

Keeping $K$ and $\tilde{K}$ smooth, M. Gage [10] and Carla Peri [15], [16] proved, independently, a stronger version of the Theorem 4.1 showing that $\mathbf{B}_{K}$ is positive on the larger interval $\left[\rho_{\text {in }}, \rho_{\text {out }}\right]$ of the minimal annulus, provided that $\tilde{K}$ is symmetric with respect to the origin.

In this section we show that the proof can be extended to non-smooth, convex, but not strictly convex, sets. 
Theorem 4.2 Let $K$ and $\tilde{K}$ be convex polygons with corresponding parallel sides, $n>4$, with $\tilde{K}$ symmetric with respect to the origin. Then there is an annulus of center $O$, inner radius $\rho_{\text {in }}$ and outer radius $\rho_{\text {out }}$ such that $\mathbf{B}_{K}(\rho) \geq 0$ for all $\rho \in\left[\rho_{\text {in }}, \rho_{\text {out }}\right]$.

Moreover, $\mathbf{B}_{K}\left(\rho_{\text {in }}\right)=\mathbf{B}_{K}\left(\rho_{\text {out }}\right)=0$ if and only if $K$ is a homothetic transformation of $\tilde{K}$.

Remark. The restriction $n>4$ is necessary since, if $\tilde{K}$ is a parallelogram $(n=4)$, then $\mathbf{B}_{K}\left(\rho_{\text {in }}\right)=\mathbf{B}_{K}\left(\rho_{\text {out }}\right)=0$ for any parallelogram $K$ whose sides are parallel to $\tilde{K}$. It turns out that this happens because the set of normal directions, non-parallel to the ones in which either $\rho_{\text {in }}$ or $\rho_{\text {out }}$ is reached, has measure zero.

To prove Theorem 4.2 we will distinguish between the case of a centrally symmetric $K$ and the one of $K$ non-symmetric with respect to the origin. The last case will require a symmetrization process as in [10] and [16], which we describe below.

Lemma 4.1 Let $K$ and $\tilde{K}$ be two convex bodies non-homothetic to each other and let $O$ be the center of the minimal annulus of $K$ relative to $\tilde{K}$. Assume that $\tilde{K}$ is symmetric with respect to $O$, while $K$ is centrally non-symmetric. Then there exists a sequence of points $A, B, C, D$ with $A, B \in \partial K \cap \partial\left(O+\rho_{\text {in }} \tilde{K}\right)$, $C, D \in \partial K \cap \partial\left(O+\rho_{\text {out }} \tilde{K}\right)$ and $0 \leq \vartheta_{A}<\vartheta_{C}<\vartheta_{B}<\vartheta_{D}<2 \pi$, where $\vartheta_{A}, \vartheta_{B}, \vartheta_{C}, \vartheta_{D}$ are the angles which correspond to $A, B, C, D$, and there is a line $\ell$ separating the points of each pair $(A, B),(C, D)$ (unless a pair lies on the line) such that $O$ is the midpoint of the segment $\ell \cap K$.

Proof. The existence of four succesive points on the boundary of $K$ which alternately touch the inner and outer boundary of the minimal annulus is proven, by different methods, in [10] and [16].

Let

$$
g(\vartheta)=\|X(\vartheta)\|-\|X(\vartheta+\pi)\|,
$$

where $X(\vartheta)$ is the position function of $K$ in the direction $(\cos \vartheta, \sin \vartheta)$. Then $g$ is a continous function, of period $2 \pi$, and $g(\vartheta)=-g(\vartheta+\pi)$. Since $K$ centrally nonsymmetric, $g$ is non-identically zero. So, by the Intermediate Value Theorem, there is at least a $\vartheta_{0}$ such that $g\left(\vartheta_{0}\right)=0$. This proves the existence of at least a line $\ell$ whose midpoint is $O$. If one of the pairs lies on such a line, it is clear, by the alternating property of the points, that the other one is separated. Two points, each belonging to a different pair, cannot lie on the line since this will imply

$$
\begin{aligned}
0 & =\rho_{\text {in }}\|\tilde{X}(\vartheta)\|-\rho_{\text {out }}\|\tilde{X}(\vartheta+\pi)\| \\
& =\left(\rho_{\text {in }}-\rho_{\text {out }}\right)\|\tilde{X}(\vartheta)\|,
\end{aligned}
$$

where $\tilde{X}$ is the position function of $\tilde{K}$, contradicting $K$ non-homothetic to $\tilde{K}$. 
However, if only one point, for example $A$, belongs to $\ell$ we distinguish between two possibilities. First, if $\ell$ separates the pair $(C, D)$, we redenote by $B$ the point on $\partial K$ determined by $\vartheta_{A}+\pi$. (This point will also be in $\partial K \cap \partial\left(O+\rho_{\text {in }} \tilde{K}\right)$ because $g\left(\vartheta_{A}\right)=0$ implies $X\left(\vartheta_{A}+\pi\right)=\rho_{\text {in }} \tilde{X}\left(\vartheta_{A}+\pi\right)$.) If $\ell$ does not separate $C$ and $D$, all the points concentrate in the same half-plane determined by $\ell$. On the other hand, since $B \in \partial K \cap \partial\left(O+\rho_{\text {in }} \tilde{K}\right)$ and $C, D \in \partial K \cap \partial\left(O+\rho_{\text {out }} \tilde{K}\right)$, we have $g\left(\vartheta_{B}\right) \leq 0, g\left(\vartheta_{C}\right) \geq 0$, and $g\left(\vartheta_{D}\right) \geq 0$. Either one of $g\left(\vartheta_{B}\right)=0$ or $g\left(\vartheta_{C}\right)=0$ will reduce the problem to the first case. For example, if $g\left(\vartheta_{C}\right)=0$ we redenote by $D$ the point on $\partial K$ determined by $\vartheta_{C}+\pi$ and the line passing through $C$ and $D$ will do it. If $g\left(\vartheta_{B}\right)<0$ and $g\left(\vartheta_{C}\right)>0$ we apply again the Intermediate Value Theorem to obtain a line with all the required properties.

Assuming that no point of the sequence lies on a line whose intersection with $K$ has the midpoint $O$, the last case left is when $g\left(\vartheta_{A}\right)$ and $g\left(\vartheta_{B}\right)$ are strictly negative, while $g\left(\vartheta_{C}\right)$ and $g\left(\vartheta_{D}\right)$ are strictly positive. Therefore $g$ changes its sign at least four times along the unit circle.

So, by the Intermediate Value Theorem, there is a sequence of, at least, four $\vartheta_{0}$ 's on the unit circle such that $g\left(\vartheta_{0}\right)=0$. This proves the existence of at least two lines whose intersection with the convex body $K$ are segments with the midpoint $O$, and they separate two consecutive points. We will show that one of the lines will satisfy the conclusion of the lemma.

Consider the arc $A B$ along the boundary of $K$ for which the angle $\angle A O B$ is smaller than $\pi$. ( $\angle A O B$ cannot be equal to $\pi$, as this will imply $g\left(\vartheta_{A}\right)=0$.) We may assume without any loss of generality that this the $\operatorname{arc} A C B$. Then will consider the line as above which separates $A$ and $C$. The points $B$ and $C$ will then lie on the boundary of $K$ situated in the same halfplane determined by the line. If the line separates $D$ from $B$, we are done. If $D$ lies in the same halfplane as $B$ and $C$, then $\angle D O C$ (whose intersection with $\partial K$ is the arc $D B C$ ) is smaller than $\pi$, so we consider the line separating $B$ and $C$. This line will strictly separate the points of each pair $(A, B),(C, D)$.

If $\ell$ is the line as in the lemma 4.1 we can consider separately the left and the right parts of $K$ determined by $\ell$ and reflect them through the center of the minimal annulus, $O$, to obtain new centrally symmetric figures $K^{\text {left }}$ and $K^{\text {right }}$. One of them may not be convex, but by considering its convex hull and keeping the same notation for the convexification, the inradius and the outradius stay the same by the separation property of the pairs of points $(A, B),(C, D)$. Notice that, since $K$ is convex, at most one of $K^{\text {left }}$ and $K^{\text {right }}$ needs to be convexified after the symmetrization process.

On the other hand, the convexification applied to a body increases the area of the body, but decreases its length. Therefore we have:

$$
\begin{gathered}
2 \mathbf{L} \geq V\left(K^{\text {left }}, \tilde{K}\right)+V\left(K^{\text {right }}, \tilde{K}\right) \\
2 \mathbf{A} \leq V\left(K^{\text {left }}, K^{\text {left }}\right)+V\left(K^{\text {right }}, K^{\text {right }}\right)
\end{gathered}
$$

which implies further

$$
2 \mathbf{B}_{K}(\rho) \geq \mathbf{B}_{K}^{\text {left }}(\rho)+\mathbf{B}_{K} \text { right }(\rho) .
$$


The symmetrization procedure applied to a convex body $K$ :
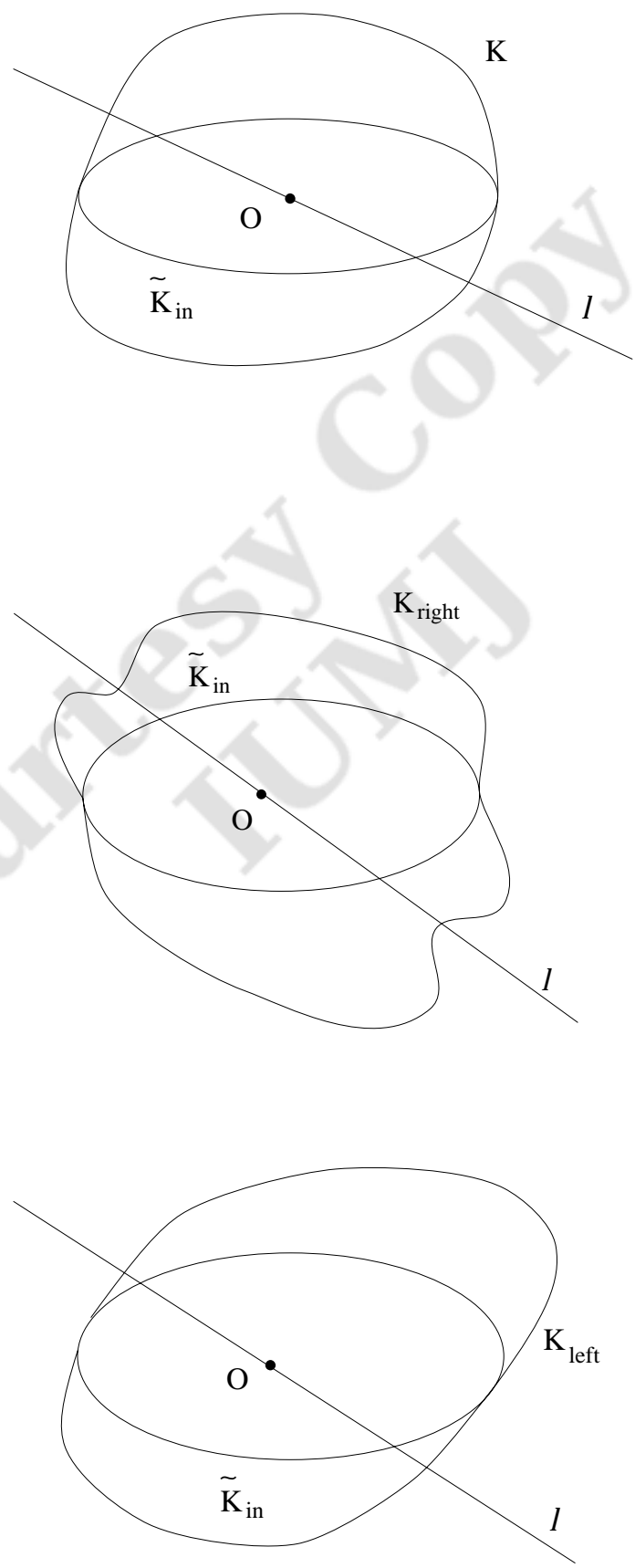
Proof of Theorem 4.2. We'll do the proof for a larger class of convex bodies, $(K, \tilde{K})$, whose boundaries have corresponding parallel flat sides, and parts of strictly positive finite curvature.

Assume that $K$ and $\tilde{K}$ are not homothetic transformations of each other and choose the minimal annulus of $K$ relative to $\tilde{K}$. We will show that, at least, one of the following $\mathbf{B}_{K}\left(\rho_{\text {in }}\right)$ or $\mathbf{B}_{K}\left(\rho_{\text {out }}\right)$ is strictly positive.

Case 1: $K$ is symmetric with respect to the origin. We use the fact that $\mathbf{B}_{K}(\rho)=m_{4}+2 m_{6}+3 m_{8}+\ldots$, for $\rho \in\left[\rho_{\text {in }}, \rho_{\text {out }}\right]$, where $m_{i}$ is the measure of the set of positions obtained by translations of $\bar{K}=\rho \tilde{K}$ in which the translate of $\partial \bar{K}$ has $i$ points in common with $\partial K$. ( The proof that Flanders gives in [6] can be easily adapted to prove this result.)

Note that, since transverse intersection points are preserved for an open set of translations, $m_{i}$ will be strictly positive if and only if there is a translation $\tau$ of $\bar{K}$ such that $\tau(\partial \bar{K})$ has $i$ transverse intersection points with $\partial K$.

Let $\tilde{K}_{\text {in }}=\rho_{\text {in }} \tilde{K}$ and $\tilde{K}_{\text {out }}=\rho_{\text {out }} \tilde{K}$, such that the minimal annulus of $K$ relative to $\tilde{K}$ is $\tilde{K}_{\text {out }}-\tilde{K}_{\text {in }}$, so $\partial K \subseteq \tilde{K}_{\text {out }}-\tilde{K}_{\text {in }}$. Recall that $\partial K$ has, at least, four intersection points with the minimal annulus such that if $A, B$ are the intersection points of $\partial K$ with $\partial \tilde{K}_{\text {in }}$ and $C, D$ are the intersection points of $\partial K$ with $\partial \tilde{K}_{\text {out }}$, then $0 \leq \vartheta_{A}<\vartheta_{C}<\vartheta_{B}<\vartheta_{D}<2 \pi$, (eventually by a switch of notation between $A$ and $B$ ) where $\vartheta_{A}, \vartheta_{B}, \vartheta_{C}, \vartheta_{D}$ are the angles which correspond to $A, B, C, D$.

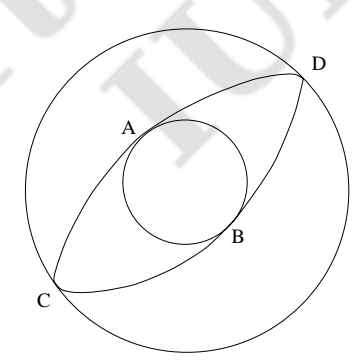

The outline of the proof is the following: we'll jiggle the inradius figure to obtain four transverse intersection points. If this fails, which occurs only when $K$ is the Minkowski sum of a line and $\tilde{K}_{\text {in }}$, we consider the outradius figure. If it can not be jiggled to obtain four transverse intersection points, then $K$ is the Minkowski sum of a line and $\tilde{K}_{\text {out }}$. If both possibilities fail, we show that $K$ is the Minkowski sum of two lines and is, therefore, a parallelogram.

To verify that $n=4$ is indeed an exception, one can consider the case of $K$ a rectangle and $\tilde{K}$ a square. No translation of $\tilde{K}_{\text {in }}$ (or $\tilde{K}_{\text {out }}$ ) can produce more than two transverse intersection points. Therefore $m_{i}=0$ for all $i$ 's greater than four.

By the symmetry assumption on $K$, we may assume without any loss of generality, that $A$ and $B$ are the tangency points of $K$ with $\tilde{K}_{\text {in }}$ such that the tangent lines to $K$ at $A$ and, respectively, $B$ are parallel. If both $A$ and $B$ are 
achieved on flat sides, consider first a translation, $\tau$, of $\tilde{K}_{\text {in }}$ along the tangents at $A, B$, until $\tau(A B)$ touches the arc $A B$ of $K$ in a point $A^{\prime}$. Assume that there exists a point $a$ on $\tau(A B)$ strictly interior to $K$, which, without any loss of generality, we can take it to be on the $\operatorname{arc} \tau(A) A^{\prime}$. Let $b$ be a point on the other $\operatorname{arc} \tau(A B)$ of $\tilde{K}_{\text {in }}$. Then a small translation in the normal direction to $\tilde{K}_{\text {in }}$ at $A^{\prime}$, combined with a small translation in the normal direction to $\tilde{K}_{\text {in }}$ at $\tau(A)$ will take $\tau(A)$ and $A^{\prime}$ in the exterior of $K$, while the images of $a$ and $b$ belong to the interior of $K$. If there does not exist any $a$ as above, i.e. the arc $\tau(A B)$ of $\tau\left(\tilde{K}_{\text {in }}\right)$ is congruent to the $\operatorname{arc} A B$ of $K$, and similarly for a translation of $\tilde{K}_{\text {in }}$ along the tangents at $A$ and $B$ in the opposite direction, then $K$ is the Minkowski sum of $\tilde{K}_{\text {in }}$ with a non-trivial line. Recall here that $K$ and $\tilde{K}$ are not homothetic translations of each other.

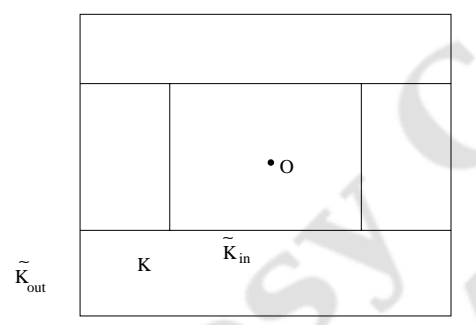

Next consider $K$ and $\tilde{K}_{\text {out }}$. They will have two opposite tangency points, that we'll call $C$ and $D$. If the whole situation repeats for $\tilde{K}_{\text {out }}$ and $\mathbf{B}_{K}\left(\rho_{\text {out }}\right)=$ $0, K$ is the Minkowski sum of $\tilde{K}_{\text {out }}$ with a non-trivial line. So assume that $K=\tilde{K}_{\text {in }}+\ell_{1}=\tilde{K}_{\text {out }}+\ell_{2}$, with $\ell_{1} \neq \ell_{2}$. First, this cannot happen if $K$ has points of strictly positive, finite curvature, since the curvature of $K$ at such a point will be simultaneously equal to the multiples of $\rho_{\text {in }}$ and $\rho_{\text {out }}$ of the curvature of $\tilde{K}$ at some point. So $K$ must be purely polygonal. On the other hand, the two equalities imply, successively, that $n-2$ flat sides of $K$ are equal to the corresponding $n-2$ flat sides of $\tilde{K}_{\text {in }}$, while $n-2$ flat sides of $K$, other than the first, are equal to the corresponding $n-2$ flat sides of $\tilde{K}_{\text {out }}$. The only possibility for this to happen is for $K$ to be a parallelogram $(n=4)$.

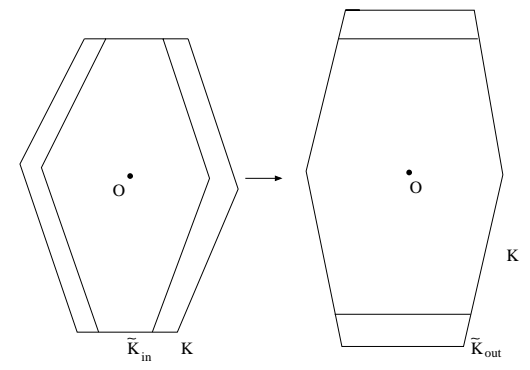

If both $A$ and $B$ are reached on curved sides then, since we have assumed $K$ and $\tilde{K}$ are not homothetic to each other, there exists the points $a$ and $b$ as 
above, so that a small translation of $\tilde{K}$ in the tangent direction to $A$ and $B$ will produce four intersection points by taking $A$ and $B$ outside $K$ and keep $a$ and $b$ inside $K$. Thus, both $\mathbf{B}_{K}\left(\rho_{\text {in }}\right)$ and $\mathbf{B}_{K}\left(\rho_{\text {out }}\right)$ are strictly positive.

Case 2: $K$ is non-symmetric with respect to the origin.

We consider the symmetrization process described earlier to obtain two centrally symmetric convex bodies $K^{\text {left }}$ and $K^{\text {right }}$. Recall from their construction that $2 \mathbf{B}_{K}(\rho) \geq \mathbf{B}_{K}$ left $(\rho)+\mathbf{B}_{K}$ right $(\rho)$, which, given the first case, leads to the positivity of $\mathbf{B}_{K}$ on $\left[\rho_{\text {in }}, \rho_{\text {out }}\right]$.

So assuming $\mathbf{B}_{K}\left(\rho_{\text {in }}\right)=\mathbf{B}_{K}\left(\rho_{\text {out }}\right)=0$, it implies

$$
\mathbf{B}_{K} \text { left }\left(\rho_{\text {in }}\right)=\mathbf{B}_{K} \text { left }\left(\rho_{\text {out }}\right)=0
$$

and

$$
\mathbf{B}_{K}^{\text {right }}\left(\rho_{\text {in }}\right)=\mathbf{B}_{K} \text { right }\left(\rho_{\text {out }}\right)=0,
$$

and therefore, by the previous case, both $K^{\text {left }}$ and $K^{\text {right }}$ are homotheties of $\tilde{K}$.

Consider first the situation when both $K^{\text {left }}$ and $K^{\text {right }}$ are obtained strictly by the symmetrization process (and therefore none of them is actually the convex hull of the symmetrized left or, respectively, right part of $K$ ). Then the segment determined by the line $\ell$ and its intersection with $\partial K$, it is identical to both $\ell \cap K^{\text {left }}$ and $\ell \cap K^{\text {right }}$. It follows that $K^{\text {left }}$ and $K^{\text {right }}$ are homotheties of $\tilde{K}$, of the same homothetic factor, and thus $K$ homothetic to $\tilde{K}$. Actually it implies further that $K$ symmetric with respect to the origin, contradicting the assumption of this case. The possibility of the polygonal case $n=4$ was ruled out, since the symmetrization process left $\tilde{K}$ unchanged, therefore its number of sides stays fixed and, by hypothesis, is greater than four.

If one of $K^{\text {left }}$ and $K^{\text {right }}$ is the convex hull of the corresponding symmetrized part of $K$, we will distinguish between a purely polygonal $K$ and one which combines flat and curved sides. Assume without any loss of generality that $K^{\text {right }}$ is obtained by the convexification applied to the symmetric body $K^{\text {right }}$ and it is relabeled the same for simplicity. In the purely polygonal subcase, $\tilde{K}$ and $K^{\text {right }}$ will have, at least, one non-parallel side. This will contradict the fact that $\tilde{K}$ is homothetic to $K^{\text {right }}$. In the other subcase, in which $\partial K$ includes curved parts, consider $\ell \cap K^{\text {left }}$ and $\ell \cap K^{\text {right }}$. Due to the convexification of $K^{\text {right }}$, these segments do not have equal length. On the other hand, $\tilde{K}$ is homothetic to both $K^{\text {left }}$ and $K^{\text {right }}$, so $\ell \cap K^{\text {left }} \neq \ell \cap K^{\text {right }}$ can only imply that $K^{\text {left }}$ and $K^{\text {right }}$ are homotheties of $\tilde{K}$ of different factors.

We will now use the property of $\ell$ to separate the points of each pair $(A, B),(C, D)$, unless one lies on the line. Assume without any loss of generality that the pair $(C, D)$ does not lie on the line and that $C$ belongs to $K^{\text {left }}$, while $D$ belongs to $K^{\text {right }}$. We have distance $(O, C)=\operatorname{distance}(O, D)=\rho_{\text {out }}$ and each of them determines the homothety factor of $K^{\text {left }}$ and, respectively, $K^{\text {right }}$ relative to $\tilde{K}$ contradicting that $K^{\text {left }}$ and $K^{\text {right }}$ are different homotheties applied to $\tilde{K}$. Therefore, none of the latter cases can happen. 
We can conclude that for a centrally non-symmetric $K$, at least one of $\mathbf{B}_{K}\left(\rho_{\text {in }}\right)$ and $\mathbf{B}_{K}\left(\rho_{\text {out }}\right)$ is strictly positive and therefore the only possibility for $K$ to have $\mathbf{B}_{K}\left(\rho_{\text {in }}\right)=\mathbf{B}_{K}\left(\rho_{\text {out }}\right)=0$ is when $K$ is a homothetic transformation of $\tilde{K}$.

We are now ready to present the proof of the Theorem 1.1.

Proof of Theorem 1.1. Notice that a self-similar solution, $\partial K$, to the system of ordinary differential equations (1) has constant isoperimetric ratio, $\mathbf{L}^{2} / \mathbf{A}$.

This implies $\delta(K)=0$ (Lemma 3.3 and Theorem 4.2), which leads to the Bonnesen functional of $K$ identically zero on $\left[\rho_{\text {in }}, \rho_{\text {out }}\right]$. By the previous theorem, $\mathbf{B}_{K}\left(\rho_{\text {in }}\right)=\mathbf{B}_{K}\left(\rho_{\text {out }}\right)=0$ implies $K$ homothetic to $\tilde{K}$.

The character of attractor of the self-similar solution follows now by showing that $\delta(K) \rightarrow 0$ as $t \rightarrow T$, since $\delta($.$) is continous in the Hausdorff metric.$

Using the inequality (12) and the CBS-inequality (7) we have:

$$
\begin{aligned}
\delta(K) \leq & 1-\frac{\alpha}{\mathbf{A L}} \sum_{i=1}^{n}\left(\frac{h_{i}}{\tilde{h}_{i}}\right)^{2} \tilde{h}_{i} \ell_{i} \\
= & \frac{\sum_{i=1}^{n}\left(\frac{h_{i}}{\tilde{h}_{i}}\right)^{2} \tilde{h}_{i} \ell_{i}}{\mathbf{L}^{2}}\left\{\frac{\mathbf{L}^{2}}{\sum_{i=1}^{n}\left(\frac{h_{i}}{\tilde{h}_{i}}\right)^{2} \tilde{h}_{i} \ell_{i}}-\frac{\alpha \mathbf{L}}{\mathbf{A}}\right\} \\
& \sum_{i=1}^{n}\left(\frac{h_{i}}{\tilde{h}_{i}}\right)^{2} \tilde{h}_{i} l_{i} \\
\mathbf{L}^{3} & \left.\mathbf{L} \sum_{i=1}^{n}\left(\frac{\tilde{\ell}_{i}}{l_{i}}\right)^{2} \tilde{h}_{i} \ell_{i}-\frac{\mathbf{L}^{2} \alpha}{\mathbf{A}}\right\}
\end{aligned}
$$

On the other hand, since $\mathbf{L}^{2} / \mathbf{A}$ decreases (Proposition 3.1) we have

$$
\frac{\sum_{i=1}^{n}\left(\frac{h_{i}}{\tilde{h}_{i}}\right)^{2} \tilde{h}_{i} \ell_{i}}{\mathbf{L}^{3}}
$$

bounded away from zero:

$$
\frac{\sum_{i=1}^{n}\left(\frac{h_{i}}{\tilde{h}_{i}}\right)^{2} \tilde{h}_{i} \ell_{i}}{\mathbf{L}^{3}} \geq \frac{1}{\mathbf{L}^{3}} \cdot \frac{\left(\sum_{i=1}^{n} h_{i} \ell_{i}\right)^{2}}{\sum_{i=1}^{n} \tilde{h}_{i} \ell_{i}}=\frac{4 \mathbf{A}^{2}}{\mathbf{L}^{4}} \geq 4\left(\frac{\mathbf{A}}{\mathbf{L}^{2}}\right)_{\left.\right|_{t=0}}^{2}>0 .
$$

From the inequality (14) we may now conclude by Lemma 3.2 that the shape of any solution $\partial K$ to the flow approaches the shape of the self-similar solution as $t \rightarrow T$. 
Acknowledgments. I would like to thank Professor Michael Gage, my advisor, for his help, suggestions and support while I was engaged in this project. I am also grateful for the referee's comments and suggestions.

\section{REFERENCES}

[1] S. Angenent, M. Gurtin, Multiphase Thermomechanics with Interfacial Structure 2. Evolution of an isothermal Interface, Archive for Rat. Mech. and Anal., 108 (1989), 323-391.

[2] W. Blaschke, Integralgeometrie, XI: Variationrechung, Abh. Math. Sem. Univ. Hasiscen 11 (1936), 359-366.

[3] T. Bonnesen, Les Problemes des isoperimetres et des isepiphanes, Gauthiers-Villars, Paris, 1929.

[4] T. Bonnesen and W. Fenchel, Theory of Convex Bodies (translated from German). Moscow, Idaho, 1987.

[5] C. Dohmen, Y. Giga, N. Mizoguchi, Existence of Selfsimilar Shrinking Curves for Anisotropic Curvature Flow Equations, Calc. Var. (to appear).

[6] H. Flanders, A proof of Minkowski's inequality for convex curves, Amer. Math. Monthly 75 (1968), 581-593.

[7] T. Fukui And Y. Giga, Motion of a graph by nonsmooth weighted curvature, Proc. of the First World Congress of Nonlinear Analysts (to appear).

[8] M. GaGe, Curve shortening makes convex curves circular, Invent. Math. 76 (1984), 357-364.

[9] M. GAGE, Evolving plane curves by curvature in relative geometries, Duke Math. Journal 72 (1993), 441-466.

[10] M. Gage, Positive centers and the Bonnesen inequality, Proc. Amer. Math. Soc. 110 (1990), 1041-1048.

[11] M. Gage and R. Hamilton, The shrinking of convex plane curves by the heat equation, J. Diff. Geometry 23 (1986), 69-96.

[12] M. Gage AND Y. LI, Evolving plane curves by curvature in relative geometries II, Duke Math. Journal, 75 (1994), 79-98.

[13] M. Grayson, The heat equation shrinks embedded plane curves to points, J. Diff. Geometry 26 (1987), 285-314.

[14] M. GuRTin, Thermomechanics of Evolving Phase Boundaries in the Plane, Clarendon Press, Oxford, 1993.

[15] C. PERI, On the minimal convex shell of a convex body, Canad. Math. Bull. 36 (1993), 466-472.

[16] C. Peri and J. M. Wills And A. Zucco, On Blachke's extension of Bonnesen's inequality, Geom. Dedicata 48 (1993), 349-357.

[17] A. Stancu, Motion of Curves Minimizing Energies with Piecewise Smooth Wulff Shapes, in preparation.

[18] J. E. TAYLOR, Constructions and conjectures in crystalline nondifferential geometry, Proceedings of the Conference in Differential Geometry, Rio de Janeiro, 1988, 'Differential Geometry,' pp. 321-336 (Pitman Monographs Surveys Pure Appl. Math. 52, Longman, Harlow, 1991).

[19] J. E. TAYLOR, Motion of curves by crystalline curvature, including triple junctions and boundary points, Diff. Geom.: Partial Diff. Eqs. on Manifolds (Los Angeles, CA, 1990), pp. 417-438, Proc. Sympos. Pure Math., Vol. 54, Part 1, AMS, Providence, RI, 1993. 
A. Stancu

Department of Mathematics

University of Rochester

Rochester, New York 14627

E-MAIL: stancu@gauss.math.rochester.edu

Received: January 18th, 1996; revised: December 1st, 1996. 